\title{
Nonlinear water waves (KdV) equation and Painlevé technique
}

\author{
Attia .A.H Mostafa \\ Faculty of Mathematics, University of Belgrade \\ Studentski trg 16, P. P. 550, 11000 Belgrade, Serbia \\ E-mail: attia716@gmail.com
}

Copyright (C)2015 Attia A.H Mostafa. This is an open access article distributed under the Creative Commons Attribution License, which permits unrestricted use, distribution, and reproduction in any medium, provided the original work is properly cited.

\begin{abstract}
The Korteweg-de Vries (KdV) equation which is the third order nonlinear PDE has been of interest since Scott Russell (1844) [5]. In this paper we study this kind of equation by Painlevé equation and through this study, we find that KdV equation satisfies Painlevé property [4], but we could not find a solution directly, so we transformed the $\mathrm{KdV}$ equation to the like-KdV equation, therefore, we were able to find four exact solutions to the original KdV equation.
\end{abstract}

Keywords: Kortewege-de Vrise equation; Painlevé property; Resonance points; Exact solutions.

\section{Introduction}

Most phenomena in the scientific field and other domains can be described and classified as nonlinear diffusion equation which normally results from natural phenomena that appear in our daily lives such as the water waves at the beach caused by wind or tides, also the movement of a ship, or those waves created by throwing a stone in a pond or by raindrops; the same applies to other physical and mathematical phenomena [2] [6]. In this study, we tried to find a solution to this type of equations although it is normally very difficult to find a clear-cut solution [3]. However, through the use of Painlevé technique it is possible to find an analytic solution which physicists, meteorologists, oceanographers, and others may benefit from in order to explain the result solution and arrive at a better understanding.

\section{Painlevé property}

In this section we apply Painlevé's property in the KdV equation:

$u_{t}+\alpha u u_{x}+u_{x x x}=0, \quad \alpha \in \mathbb{R} \backslash\{0\}$

Let $u=\sum_{j=0}^{\infty} u_{j} \phi^{j-p}$ be the series solution of the equation (1), where $\phi$ and $u_{j}$ are analytic functions in a neighbourhood of the manifold $\phi=\phi(t, x)=0$. First, we need to find value of $p$, where $p$ is the equilibrium point in the series solution. Now, to derive $u$ in the series, where $u_{t}(t, x)=\frac{\partial u(t, x)}{\partial t}, u_{x}(t, x)=\frac{\partial u(t, x)}{\partial x}$ and 
$u_{x x x}(t, x)=\frac{\partial^{3} u(t, x)}{\partial x^{3}}$, by substituting them into the equation (1), and by comparing the lowest powers in the produced equation, we find $p=2$, by associating the summation [7], we get:

$$
\begin{aligned}
& \sum_{j=3}^{\infty} u_{j-3, t} \phi^{j-5}+\alpha \sum_{j=0}^{\infty}\left[\sum_{k=0}^{j-1} u_{k} u_{j-1-k, x}+\sum_{i=0}^{j} u_{j-i} u_{i}(i-2) \phi_{x}\right] \phi^{j-5} \\
& +\sum_{j=3}^{\infty} u_{j-3, x x x} \phi^{j-5}+\sum_{j=2}^{\infty} 3(j-4) u_{j-2, x x} \phi_{x} \phi^{j-5} \\
& +\sum_{j=1}^{\infty} 3(j-3)(j-4) u_{j-1, x} \phi_{x}^{2} \phi^{j-5}+\sum_{j=2}^{\infty}(j-4) u_{j-2} \phi_{x x x} \phi^{j-5} \\
& +\sum_{j=1}^{\infty} 3(j-3)(j-4) u_{j-1} \phi_{x} \phi_{x x} \phi^{j-5}+\sum_{j=2}^{\infty} 3(j-4) u_{j-2, x} \phi_{x x} \phi^{j-5} \\
& +\sum_{j=2}^{\infty}(j-4) u_{j-2} \phi_{t} \phi^{j-5}+\sum_{j=0}^{\infty}(j-2)(j-3)(j-4) u_{j} \phi_{x}^{3} \phi^{j-5}=0
\end{aligned}
$$

To find $u_{0}$, then at $j=0$ in the equation (2), we get:

$u_{0}=-\frac{12}{\alpha} \phi_{x}^{2}$

To find $u_{1}$, then at $j=1$ in the equation (2), we get:

$u_{1}=\frac{12}{\alpha} \phi_{x x}$

To find $u_{2}$, then at $j=2$ in the equation (2), we get:

$u_{2}=-\frac{1}{\alpha} \frac{\phi_{t}}{\phi_{x}}-\frac{4}{\alpha} \frac{\phi_{x x x}}{\phi_{x}}+\frac{3}{\alpha}\left(\frac{\phi_{x x}}{\phi_{x}}\right)^{2}$,

Since $p=2$, by using the technique of truncation, and let $u_{j}=0$, for all $j>2$.

Then the series solution $u=\sum_{j=0}^{\infty} u_{j} \phi^{j-p}$, becomes:

$u=\frac{u_{0}}{\phi^{2}}+\frac{u_{1}}{\phi}+u_{2}$,

This is the relation between $u$ and $u_{2}$.

Now, in the equation (2), we have to find all coefficients of $u_{j}$, where $u_{j} \equiv 0$ for all $j<0$.

If $: \quad i=0 \Rightarrow \alpha \sum_{i=0}^{j} u_{j-i} u_{i}(i-2) \phi_{x}=2 \alpha \phi_{x}^{3} u_{j}$

and,

If $: \quad i=j \Rightarrow \alpha \sum_{i=0}^{j} u_{j-i} u_{i}(i-2) \phi_{x}=-\alpha \phi_{x}^{3}(j-2) u_{j}$.

Thus, the recursion relation is:

$$
\begin{aligned}
& (j-4)\left[j^{2}-5 j+(6-\alpha)\right] \phi_{x}^{3} u_{j}=-u_{j-3, t}-(j-4) u_{j-2} \phi_{t}-3(j-4) u_{j-2, x} \phi_{x x} \\
& -\alpha \sum_{i=1}^{j-1} u_{j-i} u_{i}(i-2) \phi_{x}-\alpha \sum_{k=0}^{j-1} u_{k} u_{j-1-k, x}-u_{j-3, x x x}-3(j-4) u_{j-2, x x} \phi_{x} \\
& -3(j-3)(j-4) u_{j-1, x} \phi_{x}^{2}-(j-4) u_{j-2} \phi_{x x x}-3(j-3)(j-4) u_{j-1} \phi_{x} \phi_{x x},
\end{aligned}
$$

We note that the coefficients of $u_{j}$ in the equation $(7)$ are $(j-4)$ and $\left[j^{2}-5 j+(6-\alpha)\right]$, then, in the general of the integer resonance point is $j=4$. The other resonance points depend on the value of $\alpha$. For example, if $\alpha=6$, the 
resonance points are $j=0,4,5$, and if $\alpha=12$, the resonance points are $j=-1,4,6$.

Now at $j=3$ in the equation (7) and using the equations (3) and (4), we have,

$u_{3}=\frac{1}{\alpha} \frac{\phi_{x t}}{\phi_{x}^{2}}+\frac{\phi_{x x} u_{2}}{\phi_{x}^{2}}+\frac{1}{\alpha} \frac{\phi_{x x x x}}{\phi_{x}^{2}}$,

When $j=4$ in the equation (7), since $u_{j}=0$ for all $j>2$, we get $u_{4}=0$.

Then the equation (1) satisfies the Painlevé property [7].

When $j=5$ in the equation (7), since $u_{j}=0$ for all $j>2$, we get,

$u_{2, t}+\alpha u_{2} u_{2, x}+u_{2, x x x}=0$.

Then $u_{2}$ is also a solution of the KdV equation (1).

\section{Analytic solution}

In this section, we follow the idea to derive analytic solution. They are invariant under this transformation [1],

$H: \phi \longrightarrow \frac{a \phi+b}{c \phi+d} \quad$ where $\quad a d-b c \neq 0$.

They are the Schwartzian derivative [3],

$S(\phi)=\frac{\phi_{x x x}}{\phi_{x}}-\frac{3}{2}\left(\frac{\phi_{x x}}{\phi_{x}}\right)^{2}$,

and dimension of velocity,

$C(\phi)=-\frac{\phi_{t}}{\phi_{x}}$.

Furthermore, we define,

$L(\phi)=-\frac{\phi_{x x}}{2 \phi_{x}}$.

The relations,

$L_{t}=C L_{x}-L C_{x}+\frac{1}{2} C_{x x}$ and $L_{x}=-L^{2}-\frac{1}{2} S$.

The compatibility of $S$ and $C$ given by,

$S_{t}+C_{x x x}+2 C_{x} S+C S_{x}=0$.

Now, by using the equations (5) and (8), we obtain:

$\alpha \phi_{x} u_{3}=\frac{\phi_{x t}}{\phi_{x}}+\frac{\alpha \phi_{x x}}{\phi_{x}}\left(-\frac{1}{\alpha} \frac{\phi_{t}}{\phi_{x}}-\frac{4}{\alpha} \frac{\phi_{x x x}}{\phi_{x}}+\frac{3}{\alpha}\left(\frac{\phi_{x x}}{\phi_{x}}\right)^{2}\right)+\frac{\phi_{x x x x}}{\phi_{x}}$.

Since, $u_{j}=0$ for all $j>2$, we get:

$\frac{\phi_{t} \phi_{x x}}{\phi_{x}^{2}}-\frac{\phi_{x t}}{\phi_{x}}=\frac{\phi_{x x x x}}{\phi_{x}}-\frac{4 \phi_{x x} \phi_{x x x}}{\phi_{x}^{2}}+3\left(\frac{\phi_{x x}}{\phi_{x}}\right)^{3}$.

Then, by comparing both sides of the equation (15) with equations (10) and (11), we observe:

$C_{x}=S_{x}$

Now, by using the equations (10), (11) and (12), then the equation (5), becomes:

$u_{2}=\frac{1}{\alpha} C-\frac{4}{\alpha} S-\frac{12}{\alpha} L^{2}$. 
We derive the equation (16), to find $u_{2, t}, \alpha u_{2} u_{2, x}$ and $u_{2, x x x}$ and substitute them into the equation (9), then:

$$
\begin{aligned}
& \frac{1}{\alpha} C_{t}-\frac{4}{\alpha} S_{t}-\frac{24}{\alpha} L L_{t}-\frac{1}{\alpha} C C_{x}-\frac{4}{\alpha} C S_{x}-\frac{24}{\alpha} C L L_{x}-\frac{4}{\alpha} S C_{x}+\frac{16}{\alpha} S S_{x} \\
& +\frac{96}{\alpha} S L L_{x}-\frac{12}{\alpha} L^{2} C_{x}+\frac{48}{\alpha} L^{2} S_{x}+\frac{288}{\alpha} L^{3} L_{x}+\frac{1}{\alpha} C_{x x x}-\frac{4}{\alpha} S_{x x x} \\
& -\frac{24}{\alpha} L L_{x x x}-\frac{72}{\alpha} L_{x} L_{x x}=0,
\end{aligned}
$$

To eliminate $L$, by using the relations (13), and by the equation (16) then, $L^{2} C_{x}-L^{2} S_{x}=0$ and $L S_{x x}-L C_{x x}=0$. And by using (14), then the equation (18), becomes:

$C_{t}+C_{x x x}+2 C_{x} S+C C_{x}=0$

By comparing the equations (14) with (19), and using the equation (16), we get:

$C_{t}=S_{t}$,

Then by the equations (16) and (20), we get, $C=S+K$ where $K$ is constant.

For $K=0$, we get:

$C=S$,

By substituting $C=S$ into the equation (14), we get:

$S_{t}+3 S S_{x}+S_{x x x}=0$

This is Korteweg-de Vries like equation (KdV).

\section{Exact solution}

Solution for constant $S$.

The constant functions $S= \pm 2 \lambda^{2}$ where $\lambda$ is constant, are solutions of the KdV like equation (22).

Lemma 4.1 [1] Let $\psi_{1}$ and $\psi_{2}$ be two linearly independent solutions of the equation,

$\frac{d^{2} \psi}{d z^{2}}+P(z)=0$

which are defined and holomorphic on some simply connected domain $D$ in complex plane, then $W(z)=\psi_{1}(z) / \psi_{2}(z)$ satisfies the equation,

$\{W, Z\}=2 P(z)$

Conversely, if $W(z)$ is a solution of (24) at all point of $D$, then one can find two linearly holomorphic independent solutions $\psi_{1}$ and $\psi_{2}$ of $(23)$ such that $W(z)=\psi_{1}(z) / \psi_{2}(z)$ in some neighborhood of $z_{0} \in D$.

Lemma 4.2 [3] The Schwartzian derivative is invariant under fractional linear transformation acting on the first argument, namely,

$\left\{\frac{a W+b}{c W+d} ; z\right\}=\{W ; z\} \quad$ where $\quad a d-b c \neq 0$

where $a, b, c$ and $d$ are constants. 


\section{Case A:}

For $S=-2 \lambda^{2}$, we have, $S=\{\phi, x\}=-2 \lambda^{2}$.

Hence $P(x)=-\lambda^{2}$ in $(24)$, and two linearly independent solutions are:

$\Psi_{1}=E(t) e^{\lambda x}+F(t) e^{-\lambda x} \quad, \quad \Psi_{2}=G(t) e^{\lambda x}+H(t) e^{-\lambda x}$

Therefore by Lemma 4.1 and Lemma 4.2, we obtain:

$\phi(t, x)=\frac{E(t) e^{\lambda x}+F(t) e^{-\lambda x}}{G(t) e^{\lambda x}+H(t) e^{-\lambda x}} \quad$ where $\quad E H-F G \neq 0$,

By using the equations (11) and (21), then:

$C=S=-\frac{\phi_{t}}{\phi_{x}}=-2 \lambda^{2}$,

Now, to find the equation of coefficients $E(t), F(t), G(t)$ and $H(t)$, we derive $\phi(t, x)$ in the equation (25), once respect to $t$ and once respect to $x$ and substituting them into the equation(26), we obtain:

$$
\begin{aligned}
\phi_{t} & =\frac{\left[G(t) E^{\prime}(t)-E(t) G^{\prime}(t)\right] e^{2 \lambda x}+\left[H(t) F^{\prime}(t)-F(t) H^{\prime}(t)\right] e^{-2 \lambda x}}{\left[G(t) e^{\lambda x}+H(t) e^{-\lambda x}\right]^{2}} \\
& +\frac{G(t) F^{\prime}(t)-F(t) G^{\prime}(t)+H(t) E^{\prime}(t)-E(t) H^{\prime}(t)}{\left[G(t) e^{\lambda x}+H(t) e^{-\lambda x}\right]^{2}}
\end{aligned}
$$

and,

$\phi_{x}=\frac{2 \lambda[H(t) E(t)-G(t) F(t)]}{\left[G(t) e^{\lambda x}+H(t) e^{-\lambda x}\right]^{2}}$,

Then, the equation (26) becomes:

$$
\begin{aligned}
C & =\frac{\left[G(t) E^{\prime}(t)-E(t) G^{\prime}(t)\right] e^{2 \lambda x}+\left[H(t) F^{\prime}(t)-F(t) H^{\prime}(t)\right] e^{-2 \lambda x}}{-2 \lambda[H(t) E(t)-G(t) F(t)]} \\
& +\frac{G(t) F^{\prime}(t)-F(t) G^{\prime}(t)+H(t) E^{\prime}(t)-E(t) H^{\prime}(t)}{-2 \lambda[H(t) E(t)-G(t) F(t)]}=-2 \lambda^{2} .
\end{aligned}
$$

Then,

$$
\begin{aligned}
& \left(G(t) E^{\prime}(t)-E(t) G^{\prime}(t)\right) e^{2 \lambda x}+\left(H(t) F^{\prime}(t)-F(t) H^{\prime}(t)\right) e^{-2 \lambda x}+G(t) F^{\prime}(t) \\
& -F(t) G^{\prime}(t)+H(t) E^{\prime}(t)-E(t) H^{\prime}(t)=4 \lambda^{3}(H(t) E(t)-G(t) F(t)) .
\end{aligned}
$$

This leads to a system of nonlinear ordinary differential equation in coefficients $E(t), F(t), G(t)$ and $H(t)$, then:

$$
\begin{aligned}
& G E^{\prime}-E G^{\prime}=0 \\
& H F^{\prime}-F H^{\prime}=0 \\
& \left(G F^{\prime}-F G^{\prime}\right)+\left(H E^{\prime}-E H^{\prime}\right)=4 \lambda^{3}(H E-G F)
\end{aligned}
$$

Particular solutions of (I) and (II) are:

$E(t)=A G(t) \quad$ and $\quad F(t)=B H(t)$

where $A$ and $B$ are real arbitrary constants. By substituting these into (III), we get:

$B\left(G(t) H^{\prime}(t)-H(t) G^{\prime}(t)\right)+A\left(H(t) G^{\prime}(t)-G(t) H^{\prime}(t)\right)=4 \lambda^{3} H(t) G(t)(A-B)$,

then:

$$
\frac{H^{\prime}(t)}{H(t)}-\frac{G^{\prime}(t)}{G(t)}=-4 \lambda^{3}
$$


By integrating the above, we get:

$\frac{H(t)}{G(t)}=\exp \left(-4 \lambda^{3} t\right)$

Then the equation (25), becomes:

$\phi(t, x)=\frac{A G(t) \exp (\lambda x)+B G(t) \exp \left(-4 \lambda^{3} t-\lambda x\right)}{G(t) \exp (\lambda x)+G(t) \exp \left(-4 \lambda^{3} t-\lambda x\right)}$,

Which leads to:

$$
\begin{aligned}
\phi(t, x) & =\frac{A e^{\lambda \xi_{1}}+B e^{-\lambda \xi_{1}}}{e^{\lambda \xi_{1}}+e^{-\lambda \xi_{1}}}, \quad \text { where } \xi_{1}=x+2 \lambda^{2} t \\
& =\frac{(A+B) \cosh \lambda \xi_{1}+(A-B) \sinh \lambda \xi_{1}}{2 \cosh \lambda \xi_{1}}
\end{aligned}
$$

Then:

$\phi(t, x)=K_{1}+K_{2} \tanh \lambda \xi_{1}$,

where $K_{1}$ and $K_{2}$ are arbitrary constants, and $K_{1}=(A+B) / 2$ and $K_{2}=(A-B) / 2$. For $K_{1}=0$, and by substituting the equation (27) into the equation (5), we obtain:

$$
\begin{aligned}
u_{2} & =-\frac{1}{\alpha} \frac{2 K_{2} \lambda^{3} \operatorname{sech}^{2} \lambda \xi_{1}}{K_{2} \lambda \operatorname{sech}^{2} \lambda \xi_{1}}-\frac{4}{\alpha} \frac{-2 K_{2} \lambda^{3} \operatorname{sech}^{4} \lambda \xi_{1}+4 K_{2} \lambda^{3} \operatorname{sech}^{2} \lambda \xi_{1} \tanh ^{2} \lambda \xi_{1}}{K_{2} \lambda \operatorname{sech}^{2} \lambda \xi_{1}} \\
& +\frac{3}{\alpha} \frac{4 K_{2}^{2} \lambda^{4} \operatorname{sech}^{4} \lambda \xi_{1} \tanh ^{2} \lambda \xi_{1}}{K_{2}^{2} \lambda^{2} \operatorname{sech}^{4} \lambda \xi_{1}}
\end{aligned}
$$

Then:

$u_{2}=\frac{12 \lambda^{2}}{\alpha}\left(\operatorname{sech}^{2} \lambda \xi_{1}-\frac{1}{2}\right)$, where $\xi_{1}=x+2 \lambda^{2} t$,

Hence $u_{2}(t, x)$ is the first exact solution for $\mathrm{KdV}$ equation (1). Now, by the equations (3), (4), (6) and (27), we obtain:

$$
\begin{aligned}
u & =\frac{-12}{\alpha} \frac{\phi_{x}^{2}}{\phi^{2}}+\frac{12}{\alpha} \frac{\phi_{x x}}{\phi}+u_{2} \\
& =\frac{-12}{\alpha} \frac{K_{2}^{2} \lambda^{2} \operatorname{sech}^{4} \lambda \xi_{1}}{K_{2}^{2} \tanh ^{2} \lambda \xi_{1}}-\frac{24}{\alpha} \frac{K_{2} \lambda^{2} \operatorname{sech}^{2} \lambda \xi_{1} \tanh \lambda \xi_{1}}{K_{2} \tanh \lambda \xi_{1}}+u_{2}
\end{aligned}
$$

Then:

$u=-\frac{12 \lambda^{2}}{\alpha}\left(\operatorname{csech}^{2} \lambda \xi_{1}+\frac{1}{2}\right), \quad$ where $\quad \xi_{1}=x+2 \lambda^{2} t$,

Hence $u(t, x)$ is the second exact solution for KdV equation (1).

\section{Case B:}

For $S=2 \lambda^{2}$, we have: $S=\{\phi, x\}=2 \lambda^{2}$.

Hence $P(x)=-\lambda^{2}$ in $(24)$, and two linearly independent solutions are:

$\Psi_{3}=E(t) e^{\lambda i x}+F(t) e^{-\lambda i x} \quad, \quad \Psi_{4}=G(t) e^{\lambda i x}+H(t) e^{-\lambda i x}$

Therefore, from Lemma 4.1 and Lemma 4.2, one obtains:

$\phi(t, x)=\frac{E(t) e^{\lambda i x}+F(t) e^{-\lambda i x}}{G(t) e^{\lambda i x}+H(t) e^{-\lambda i x}} \quad$ where $\quad E H-F G \neq 0$, 
By using the equations (11) and (21), then:

$C=S=-\frac{\phi_{t}}{\phi_{x}}=2 \lambda^{2}$

Now to find the equation of coefficients $E(t), F(t), G(t)$ and $H(t)$, we derive $\phi(t, x)$ in the equation (28), once respect to $t$ and once respect to $x$ and by substituting them into the equation(29), we obtain:

$$
\begin{aligned}
C & =\frac{\left[G(t) E^{\prime}(t)-E(t) G^{\prime}(t)\right] e^{2 \lambda i x}+\left[H(t) F^{\prime}(t)-F(t) H^{\prime}(t)\right] e^{-2 \lambda i x}}{-2 i \lambda[H(t) E(t)-G(t) F(t)]} \\
& +\frac{G(t) F^{\prime}(t)-F(t) G^{\prime}(t)+H(t) E^{\prime}(t)-E(t) H^{\prime}(t)}{-2 i \lambda[H(t) E(t)-G(t) F(t)]}=2 \lambda^{2} .
\end{aligned}
$$

This leads to a system of nonlinear ordinary differential equations with coefficients $E(t), F(t), G(t)$ and $H(t)$, then:

(I) $\quad G E^{\prime}-E G^{\prime}=0$,

(II) $H F^{\prime}-F H^{\prime}=0$,

(III) $\left.\quad G F^{\prime}-F G^{\prime}\right)+\left(H E^{\prime}-E H^{\prime}\right)=-4 i \lambda^{3}(H E-G F)$,

Particular solutions of (I) and (II) are:

$E(t)=M G(t) \quad$ and $\quad F(t)=N H(t)$

where $M$ and $N$ are real arbitrary constants. By substituting these into (III), we get:

$\frac{H(t)}{G(t)}=\exp \left(4 i \lambda^{3} t\right)$

Then the equation (28), becomes:

$\phi(t, x)=\frac{M G(t) \exp (\lambda i x)+N G(t) \exp \left(4 \lambda^{3} i t-\lambda i x\right)}{G(t) \exp (\lambda i x)+G(t) \exp \left(4 \lambda^{3} i t-\lambda i x\right)}$,

Which leads to:

$$
\begin{aligned}
\phi(t, x) & =\frac{M e^{\lambda i \xi_{2}}+N e^{-\lambda i \xi_{2}}}{e^{\lambda i \xi_{2}}+e^{-\lambda i \xi_{2}}}, \quad \text { where } \xi_{2}=x-2 \lambda^{2} t \\
& =\frac{(M+N) \cos \lambda \xi_{2}+(M-N) \sin \lambda \xi_{2}}{2 \cos \lambda \xi_{2}}
\end{aligned}
$$

Then:

$\phi(t, x)=K_{3}+K_{4} \tan \lambda \xi_{2}$,

where $K_{3}=(M+N) / 2$ and $K_{4}=(M-N) / 2$ are arbitrary constants. For $K_{3}=0$, by substituting the equation (30) into the equation (5), we get:

$$
\begin{aligned}
\widehat{u}_{2} & =\frac{2}{\alpha} \frac{K_{4} \lambda^{3} \sec ^{2} \lambda \xi_{2}}{K_{4} \lambda \sec ^{2} \lambda \xi_{2}}-\frac{4}{\alpha} \frac{2 K_{4} \lambda^{3} \sec ^{4} \lambda \xi_{2}+4 K_{4} \lambda^{3} \sec ^{2} \lambda \xi_{2} \tan ^{2} \lambda \xi_{2}}{K_{4} \lambda \sec ^{2} \lambda \xi_{2}} \\
& +\frac{3}{\alpha} \frac{4 K_{4}^{2} \lambda^{4} \sec ^{4} \lambda \xi_{2} \tan ^{2} \lambda \xi_{2}}{K_{4}^{2} \lambda^{2} \sec ^{4} \lambda \xi_{2}}
\end{aligned}
$$

Then:

$\widehat{u}_{2}=-\frac{12 \lambda^{2}}{\alpha}\left(\sec ^{2} \lambda \xi_{2}-\frac{1}{2}\right)$, where $\xi_{2}=x-2 \lambda^{2} t$.

Hence $\widehat{u}_{2}(t, x)$ is the third exact solution for KdV equation (1). Now, by the equations (3), (4), (6) and (30), we get:

$\widehat{u}=\frac{-12}{\alpha} \frac{\phi_{x}^{2}}{\phi^{2}}+\frac{12}{\alpha} \frac{\phi_{x x}}{\phi}+\widehat{u}_{2}$.

Then:

$\widehat{u}=-\frac{12 \lambda^{2}}{\alpha}\left(\operatorname{csec}^{2} \lambda \xi_{2}-\frac{1}{2}\right), \quad$ where $\quad \xi_{2}=x-2 \lambda^{2} t$.

Hence $\widehat{u}(t, x)$ is the fourth exact solution for $\mathrm{KdV}$ equation (1). 


\section{References}

[1] A. Alderani, "Painlevé analysis and Lie symmetries of some nonlinear PDEs", Ph.D thesis, Istanbul Technical University, Turkey (1996).

[2] A. Ali, "Finite element studies of the Korteweg-de Vries equation", Ph.D thesis in mathematics. University of Wales, $U K(1989)$.

[3] A. Mostafa, "Some solutions of the modified Korteweg-de Vries equation by Painleve test", International Proceedings of Computer Science and Information Technology, Vol.59, (2014), pp.105-111.

[4] D. Baldwin, Symbolic algorithms and software for the Painlevé test and recursion operators for nonlinear partial differential equations, master thesis, Mathematical and Computer Sciences, Golden School, Colorado, USA, (2004).

[5] K. Brauer, The KdV equation: History, exact solutions, and graphical representation, University of Osnabrück, Applied Systems Science, Germany, (2006).

[6] W. Hereman, "Shallow water waves and solitary waves", Mathematics of Complexity and Dynamical Systems, (2011), pp.1520-1532.

[7] W. Steep and N. Euler, Nonlinear Evolution Equations and Painlevé Test, World Scientific Publishing Co. Pte. Ltd, (1988). 